\title{
Parametric Equations for Space Curves Whose Spherical Images Are Slant Helices
}

\author{
Abderrazzak EL HAIMI $^{1}$, Malika IZID ${ }^{1}$ \& Amina OUAZZANI CHAHDI ${ }^{1}$ \\ ${ }^{1}$ Faculty of Sciences Ben M'Sik, University of HASSAN II Casablanca, Morrocco \\ Correspondence: Malika IZID, Faculty of Sciences Ben M’Sik, University of HASSAN II Casablanca, Morrocco. \\ E-mail: izid.malika@gmail.com
}

Received: July 10, 2019 Accepted: September 12, 2019 Online Published: September 22, 2019

doi:10.5539/jmr.v11n5p82

URL: https://doi.org/10.5539/jmr.v11n5p82

\begin{abstract}
The curve whose tangent and binormal indicatrices are slant helices is called a slant-slant helix.

In this paper, we give a new characterization of a slant-slant helix and determine a vector differential equation of the third order satisfied by the derivative of principal normal vector fields of a regular curve. In terms of solution, we determine the parametric representation of the slant-slant helix from the intrinsic equations.
\end{abstract}

Finally, we present some examples of slant-slant helices by means of intrinsic equations.

Keywords: alternative moving frame, intrinsic equations, slant-slant helix

\section{Introduction}

Helical structures are an important framework in differential geometry and it was heavily studied for a long time and is still studied. We can see them in nature, architecture, simulation of kinematic motion or design of highways and mechanic tools. A general helix is defined by the property that its tangent vector field makes a constant angle with a fixed straight line which is the axis of the general helix in Euclidean 3-space. The well-known result was stated by M.A. Lancret in 1806 and first proved by B. Saint Venant in 1845 (see D. J. Struik, 1961 \& M. A. Lancret, 1806, p. 416-454 for details). A necessary and sufficient condition for a curve to be a general helix is the fact that the ratio

$$
\frac{\tau}{\kappa}
$$

is constant along the curve, where $\kappa$ and $\tau$ denote the curvature and the torsion of the curve, respectively. If both $\kappa$ and $\tau$ are non-zero constants, then the curve is called a circular helix.

S. Izumiya \& N. Takeuchi (2004) have defined a new special curve called slant helix for which the principal normal lines make a constant angle with a fixed straight line and they characterize a slant helix if and only if the geodesic curvature

$$
\sigma=\frac{\kappa^{2}}{\left(\kappa^{2}+\tau^{2}\right)^{\frac{3}{2}}}\left(\frac{\tau}{\kappa}\right)^{\prime}
$$

of principal image of the principal normal indicatrice is a constant function. The spherical images of the Frenet vectors of a slant helix have been studied by L. Kula \& Y. Yayli (2005), and they have proved that the spherical images of a slant helix are spherical helices. Later, L. Kula, N. Ekmekci, Y. Yayli \& K. ILarslan (2010), characterize slant helices by certain differential equations verified for each one of spherical indicatrix in Euclidean 3-space.

Beyhan uzunoğlu, Ismail GÖk \& Yusuf Yayli (2013), investigated a curve whose spherical images (the tangent indicatrice and binormal indicatrix) are slant helices and called it a slant-slant helix and have given some characterizations. We obtain that the spherical images are spherical slant helices.

The problem of determination of the parametric representation of an arbitrary space curve according to the intrinsic equations is still open in the Euclidean space $E^{3}$ ( L.P. Eisenhart(1909) \& M. M. Lipschutz (1969)). In one sense it was solved by Hoppe, who reduces it to solving Riccati equations back in 1862, see P. D. Scofied(1995). However, this problem was also solved in three special cases. Firstly, in the case of a plane curve $(\tau=0)$. Secondly, in the case of helix ( $\kappa$ and $\tau$ are both non-vanishing constant). In A.T. Ali(2011) \& A.T Ali(2012), the author adapted 
fundamental existence and uniqueness theorem for space curves in Euclidean space $E^{3}$ and constructed a vector differential equation to solve this problem in the case of a general helix $\left(\frac{\tau}{\kappa}\right.$ is constant $)$ and a slant helix $\left(\frac{\kappa^{2}}{\left(\kappa^{2}+\tau^{2}\right)^{\frac{3}{2}}}\left(\frac{\tau}{\kappa}\right)^{\prime}\right.$ is constant $)$.

In this work, we use vector differential equations established by means of alternative moving frame in Euclidean space $E^{3}$ to determine position vectors of the arbitrary curves according to the standard frame in $E^{3}$. We also obtain position vectors of a slant-slant helix from intrinsic equations in $E^{3}$. Besides, we present some new characterizations of a slant-slant helix and we give some illustrative examples.

\section{Preliminaries}

In the Euclidean space $E^{3}$, it is well known that to each unit speed curve with at least four continuous derivatives, one can associate three mutually orthogonal unit vector fields $T, N$ and $B$ which are called respectively, the tangent, the principal normal and the binormal vector fields (H. H. Hacisalihoglu (2000)).

Let $\psi: I \subset \mathbb{R} \rightarrow E^{3}, \psi=\psi(s)$, be an arbitrary curve in $E^{3}$. The curve $\psi$ is said to be unit speed or parametrized by the arc-length if $\left\langle\psi^{\prime}(s), \psi^{\prime}(s)\right\rangle=1$ for any $s \in I$. In particular, if $\psi^{\prime}(s) \neq 0$ for any $s$, then it is possible to re-parameterize $\psi$, that is, $\alpha=\psi(\phi(s))$ so that $\alpha$ is parametrized by the arc-length. Thus, we will assume throughout this work that $\psi$ is a unit speed curve, where $\langle$,$\rangle is on Euclidean inner product.$

Let $\{T(s), N(s), B(s)\}$ be the Frenet moving frame along $\psi$. The Frenet equations for $\psi$ are given by D. J. Struik (1961):

$$
\left[\begin{array}{c}
T^{\prime}(s) \\
N^{\prime}(s) \\
B^{\prime}(s)
\end{array}\right]=\left[\begin{array}{ccc}
0 & \kappa(s) & 0 \\
-\kappa(s) & 0 & \tau(s) \\
0 & -\tau(s) & 0
\end{array}\right]\left[\begin{array}{c}
T(s) \\
N(s) \\
B(s)
\end{array}\right],
$$

where $\kappa$ and $\tau$ are the curvature and the torsion of $\psi$, respectively.

\section{Slant-Slant Helices and Their Characterizations}

In this section, we investigate a curve whose spherical images, (the tangent indicatrix and binormal indicatrix) are slant helices (for details see Beyhan uzunoğlu, Ismail GÖk \& Yusuf Yayli (2013)). Then we call it a slant-slant helix and we give some characterizations of it using the alternative moving frame.

Denote by $\{\mathrm{N}, \mathrm{C}, \mathrm{W}\}$ the alternative moving frame along the curve $\psi$ in Euclidean space $E^{3}$. Note that $N, C=\frac{N^{\prime}}{\left\|N^{\prime}\right\|}$ and $W=\frac{\tau T+\kappa B}{\sqrt{\tau T+\kappa B}}$ are the principal normal vector, the derivative of principal normal vector and the unit Darboux vector, respectively. For the derivatives of the alternative moving frame, we have

$$
\left[\begin{array}{c}
N^{\prime}(s) \\
C^{\prime}(s) \\
W^{\prime}(s)
\end{array}\right]=\left[\begin{array}{ccc}
0 & f(s) & 0 \\
-f(s) & 0 & g(s) \\
0 & -g(s) & 0
\end{array}\right]\left[\begin{array}{c}
N(s) \\
C(s) \\
W(s)
\end{array}\right]
$$

where $f=\sqrt{\kappa^{2}+\tau^{2}}$ and $\sigma=\sigma f$ ( $\kappa$ and $\tau$ the curvature and the torsion of the curve $\psi$, respectively). If we write this curve in another parametric representation $\psi=\psi(\theta)$, where $\theta=\int f(s) d s$, we have the new alternative moving frame equations as follows:

$$
\left[\begin{array}{c}
N^{\prime}(\theta) \\
C^{\prime}(\theta) \\
W^{\prime}(\theta)
\end{array}\right]=\left[\begin{array}{ccc}
0 & 1 & 0 \\
-1 & 0 & \sigma(\theta) \\
0 & -\sigma(\theta) & 0
\end{array}\right]\left[\begin{array}{c}
N(\theta) \\
C(\theta) \\
W(\theta)
\end{array}\right]
$$

where $\sigma(\theta)=\frac{g(\theta)}{f(\theta)}$.

Definition 3.1 Let $\psi: I \subset \mathbb{R} \rightarrow E^{3}$ be a unit speed curve in Euclidean 3-space with Frenet frame $(T, N, B)$. A curve $\psi$ is called a slant-slant helix if the vector field $C=\frac{N^{\prime}}{\left\|N^{\prime}\right\|}$ makes a constant angle $\phi$ with a fixed direction $u$, that is, 


$$
\langle C, u\rangle=\cos \phi, \phi=\text { constant }
$$

along the curve $\psi$.

Lemma 3.2 Let $\psi: I \subset \mathbb{R} \rightarrow E^{3}$ be a unit speed curve in the Euclidean 3-space. Then the curve $\psi$ is a slant-slant helix if and only if the geodesic curvature

$$
\Gamma=\frac{f^{2}}{\left(f^{2}+g^{2}\right)^{\frac{3}{2}}}\left(\frac{g}{f}\right)^{\prime}
$$

of the principal image of the vector field $C$ indicatrix is a constant function.

The following lemma give a new characterization for slant-slant helices in $E^{3}$.

Lemma 3.3 Let $\psi: I \rightarrow E^{3}$ be a curve that is parametrized by arc-length with intrinsic equations $\kappa=\kappa(s)$ and $\tau=\tau(s)$. The curve $\psi$ is a slant-slant helix (its vectors $C$ make a constant angle, $\phi= \pm \arccos (n)$, with a fixed straight line in the space) if and only if

$$
g(s)= \pm \frac{m f(s) \int f(s) d s}{\sqrt{1-m^{2}\left(\int f(s) d s\right)^{2}}}
$$

where $f(s)=\sqrt{\kappa^{2}(s)+\tau^{2}(s)}, g(s)=f(s) \sigma(s)$ and $m=\frac{n}{\sqrt{1-n^{2}}}$.

Proof. $(\Rightarrow)$ Let $U$ be a unit fixed vector makes a constant angle, $\phi= \pm \arccos (n)$, with the vector $C$. Therefore

$$
\langle C, U\rangle=n
$$

Differentiating the Eq. (5) with respect to the variable $\theta=\int f(s) d s$ and using the new alternative moving frame equations (3) we get

$$
\langle-N(\theta)+\sigma(\theta) W(\theta), U\rangle=0 \text {. }
$$

Therefore,

$$
\langle N, U\rangle=\sigma\langle W, U\rangle .
$$

If we put $\langle W, U\rangle=b$, we can write

$$
U=b \sigma N+n C+b W
$$

From the unitary of the vector $U$, we get $b= \pm \sqrt{\frac{1-n^{2}}{1+\sigma^{2}}}$. Therefore, the vector $U$ can be written as

$$
U= \pm \sigma \sqrt{\frac{1-n^{2}}{1+\sigma^{2}}} N+n C \pm \sqrt{\frac{1-n^{2}}{1+\sigma^{2}}} W
$$

If we differentiate Eq.(6) again, we obtain

$$
\left\langle\sigma^{\prime} W-\left(1+\sigma^{2}\right) C, U\right\rangle=0 .
$$

Eqs.(7) and (8) lead to the following differential equation

$$
m= \pm \frac{\sigma^{\prime}}{\left(1+\sigma^{2}\right)^{\frac{3}{2}}}
$$

where $m=\frac{n}{\sqrt{1-n^{2}}}$. Integrating the above equation, we get

$$
\frac{\sigma}{\sqrt{1+\sigma^{2}}}= \pm m\left(\theta+c_{1}\right)
$$

where $c_{1}$ is an integration constant. The integration constant can disappear with a parameter change $\theta \rightarrow \theta-c_{1}$. Solving the Eq.(9) with $\sigma$ as unknown we have

$$
\sigma= \pm \frac{m \theta}{\sqrt{1-m^{2} \theta^{2}}}
$$


Finally, $g(s)=f(s) \sigma(s)$, And that's the desired result.

$(\Longleftarrow)$ Suppose that

$$
g(s)= \pm \frac{m f(s) \int f(s) d s}{\sqrt{1-m^{2}\left(\int f(s) d s\right)^{2}}}
$$

The function $\sigma$ can be written as $\sigma(\theta)= \pm \frac{m \theta}{\sqrt{1-m^{2} \theta^{2}}}$ and let us consider the vector

$$
U=n\left(\theta N+C \pm \frac{1}{m} \sqrt{1-m^{2} \theta^{2}} W\right) .
$$

We will prove that the vector $U$ is a constant vector. Indeed, applying the alternative moving frame formula (3)

$$
\frac{d U}{d \theta}=n\left(N+\theta C-N+\sigma W \mp \frac{m \theta}{\sqrt{1-m^{2} \theta^{2}}} W \mp \frac{1}{m} \sqrt{1-m^{2} \theta^{2}} \sigma C\right)=0 .
$$

Therefore, the vector $U$ is constant and $\langle C, U\rangle=n$. This concludes the proof of Lemma 3.3.

\section{Position Vectors of Slant-Slant Helices}

In this section, we give a vector differential equation satisfied by the derivative of principal normal vector fields to determine a position vector of an arbitrary curve according to standard frame in $E^{3}$. We obtain, by using the new characterization, a position vector of a slant- slant helix from intrinsic equations.

Theorem 4.1 Let $\psi=\psi(s)$ be a unit speed curve in the Euclidean 3-space. Suppose $\psi=\psi(\theta)$ is another parametric representation of this curve by the parameter $\theta=\int f(s) d s$. Then, the vector $C$ satisfies a vector differential equation of third order as follows:

$$
\frac{1}{\sigma(\theta)}\left[\frac{1}{\sigma^{\prime}}\left(C^{\prime \prime}(\theta)+\left(1+\sigma^{2}\right) C(\theta)\right)\right]^{\prime}+C(\theta)=0
$$

ere $\sigma(\theta)=\frac{g(\theta)}{f(\theta)}$.

Proof. Let $\psi=\psi(s)$ be a unit speed curve in Euclidean 3-space. If we differentiate the second equation of the new alternative moving frame (3) and use the first and third equations, we have

$$
W=\frac{1}{\sigma^{\prime}}\left[C^{\prime \prime}+\left(1+\sigma^{2}\right) C\right] .
$$

Differentiating the above equation and using the last equation from (3), we obtain a vector differential equation of third order (11) as desired.

The equation (11) is not easy to solve in the general case. If one solves this equation, the natural representation of the position vector of an arbitrary space curve can be determined as follows:

$$
\psi(s)=\int\left(\int \kappa(s)\left(\int f(s) C(s) d s\right) d s\right) d s,
$$

or in parametric representation

$$
\psi(\theta)=\int \frac{1}{f(\theta)}\left(\int \frac{\kappa(\theta)}{f(\theta)}\left(\int C(\theta) d \theta\right) d \theta\right) d \theta
$$

where $\theta=\int f(s) d s$.

We can solve the Eq.(11) in the case of a slant-slant helix.

Theorem 4.2 The position vector $\psi=\left(\psi_{1}, \psi_{2}, \psi_{3}\right)$ of a slant-slant helix is computed in the natural representation form: 


$$
\left\{\begin{array}{l}
\psi_{1}(s)=\frac{n}{m} \int\left[\int \kappa(s)\left[\int f(s) \cos \left[\frac{1}{n} \arcsin \left(m \int f(s) d s\right)\right] d s\right] d s\right] d s, \\
\psi_{2}(s)=\frac{n}{m} \int\left[\int \kappa(s)\left[\int f(s) \sin \left[\frac{1}{n} \arcsin \left(m \int f(s) d s\right)\right] d s\right] d s\right] d s, \\
\psi_{3}(s)=n \int\left[\int \kappa(s)\left[\int f(s) d s\right] d s\right] d s,
\end{array}\right.
$$

or in the parametric form

$$
\left\{\begin{array}{l}
\psi_{1}(\theta)=\frac{n}{m} \int \frac{1}{f(\theta)}\left[\int \frac{\kappa(\theta)}{f(\theta)}\left[\int \cos \left[\frac{1}{n} \arcsin (m \theta)\right] d \theta\right] d \theta\right] d \theta, \\
\psi_{2}(\theta)=\frac{n}{m} \int \frac{1}{f(\theta)}\left[\int \frac{\kappa(\theta)}{f(\theta)}\left[\int \sin \left[\frac{1}{n} \arcsin (m \theta)\right] d \theta\right] d \theta\right] d \theta, \\
\psi_{3}(\theta)=n \int \frac{1}{f(\theta)}\left[\int \frac{\kappa(\theta)}{f(\theta)} \theta d \theta\right] d \theta,
\end{array}\right.
$$

or in the useful parametric form

$$
\left\{\begin{array}{l}
\psi_{1}(t)=\frac{n^{4}}{m^{4}} \int \frac{\cos (n t)}{f(t)}\left[\int \frac{\kappa(t)}{f(t)} \cos (n t)\left[\int \cos (t) \cos (n t) d t\right] d t\right] d t \\
\psi_{2}(t)=\frac{n^{4}}{m^{4}} \int \frac{\cos (n t)}{f(t)}\left[\int \frac{\kappa(t)}{f(t)} \cos (n t)\left[\int \sin (t) \cos (n t) d t\right] d t\right] d t \\
\psi_{3}(t)=\frac{n^{3}}{m^{3}} \int \frac{\cos (n t)}{f(t)}\left(\int \frac{\kappa(t)}{f(t)} \cos (n t) \sin (n t) d t\right) d t
\end{array}\right.
$$

where $\theta=\int f(s) d s, t=\frac{1}{n} \arcsin (m \theta), m=\frac{n}{\sqrt{1-n^{2}}}, n=\cos (\phi)$ and $\phi$ is the angle between the fixed straight line (axis of a slant-slant helix) and the vector $C$ of the curve.

Proof. If $\psi$ is a slant-slant helix whose vector $C$ makes an angle $\phi=\arccos (n)$ with a straight line $U$, then we can write $\sigma(\theta)= \pm \frac{m \theta}{\sqrt{1-m^{2} \theta^{2}}}$, where $\sigma=\frac{g}{f}, \theta=\int f(s) d s$ and $m=\frac{n}{\sqrt{1-n^{2}}}$. Therefore the Eq.(11) becomes

$$
\left(1-m^{2} \theta^{2}\right) C^{\prime \prime \prime}(\theta)-3 m^{2} \theta C^{\prime \prime}(\theta)+C^{\prime}(\theta)=0 .
$$

If we write the vector $C$ as the following:

$$
C=C_{1}(\theta) e_{1}+C_{2}(\theta) e_{2}+C_{3}(\theta) e_{3},
$$

the curve $\psi$ is a slant-slant helix, i.e. the vector $C$ makes a constant angle $\phi$, with the constant vector called the axis of the slant-slant helix, so without loss of generality, we can take the axis of a slant-slant helix parallel to $e_{3}$, then

$$
C_{3}=\left\langle C, e_{3}\right\rangle=n \text {. }
$$

On the other hand the vector $C$ is a unit vector, so the following condition is satisfied

$$
C_{1}^{2}+C_{2}^{2}=1-n^{2}=\frac{n^{2}}{m^{2}}
$$

The general solution of Eq.(21) can be written in the following form:

$$
\left\{\begin{array}{l}
C_{1}(\theta)=\frac{n}{m} \cos (\lambda(\theta)), \\
C_{2}(\theta)=\frac{n}{m} \sin (\lambda(\theta)),
\end{array}\right.
$$

where $\lambda$ is an arbitrary function of $\theta$. Every component of the vector $C$ satisfies Eq.(18). So, substituting the components $C_{1}(\theta)$ and $C_{2}(\theta)$ in the Eq.(18), we have the following differential equations of the function $\lambda(\theta)$

$$
\begin{aligned}
3 \lambda^{\prime}(\theta)\left[m^{2} \theta \lambda^{\prime}(\theta)-\left(1-m^{2} \theta^{2}\right)\right] \cos (\lambda(\theta)) & -\left[\lambda^{\prime}(\theta)-3 m^{2} \theta \lambda^{\prime \prime}(\theta)-\left(1-m^{2} \theta^{2}\right)\left(\lambda^{\prime 3}(\theta)-\lambda^{\prime \prime \prime}(\theta)\right)\right] \sin (\lambda(\theta))=0, \\
3 \lambda^{\prime}(\theta)\left[m^{2} \theta \lambda^{\prime}(\theta)-\left(1-m^{2} \theta^{2}\right)\right] \sin (\lambda(\theta)) & \\
+\left[\lambda^{\prime}(\theta)-3 m^{2} \theta \lambda^{\prime \prime}(\theta)-\left(1-m^{2} \theta^{2}\right)\left(\lambda^{\prime 3}(\theta)-\lambda^{\prime \prime \prime}(\theta)\right)\right] \cos (\lambda(\theta)) & =0 .
\end{aligned}
$$


It is easy to prove that the above two equations lead to the following two equations;

$$
\begin{gathered}
m^{2} \theta \lambda^{\prime}(\theta)-\left(1-m^{2} \theta^{2}\right) \lambda^{\prime \prime}(\theta)=0, \\
\lambda^{\prime}(\theta)-3 m^{2} \theta \lambda^{\prime \prime} \theta-\left(1-m^{2} \theta^{2}\right)\left(\lambda^{\prime 3}-\lambda^{\prime \prime \prime}(\theta)\right)=0 .
\end{gathered}
$$

The general solution of Eq. (25) is

$$
\lambda(\theta)=c_{1} \arcsin (m \theta)+c_{2},
$$

where $c_{1}$ and $c_{2}$ are the constants of integration. The constant $c_{2}$ can disappear if we change the parameter $\lambda \rightarrow \lambda+c_{2}$. Substituting the solution (27) in the Eq. (26), we obtain the following condition:

$$
c_{1} m\left(1+m^{2}\left(1-c_{1}\right)\right)=0,
$$

which leads to $c_{1}=\frac{\sqrt{1+m^{2}}}{m}=\frac{1}{n}$, where $m \neq 0$ and $c_{1} \neq 0$.

Now, the vector $C$ takes the following form:

$$
\left\{\begin{array}{l}
C_{1}(\theta)=\frac{n}{m} \cos \left(\frac{1}{n} \arcsin (m \theta)\right), \\
C_{2}(\theta)=\frac{n}{m} \sin \left(\frac{1}{n} \arcsin (m \theta)\right), \\
C_{3}(\theta)=n .
\end{array}\right.
$$

If we substitute the Eq. (28) in the Eq. (14), we have the two Eq. (15) and (16). It is easy to arrive the Eq.(17), if we take the new parameter $t=\frac{1}{n} \arcsin (m \theta)$, which completes the proof.

\section{Examples}

Example 5.1 The case of a slant-slant helix with $\quad \kappa=\frac{\mu}{m} \cos (\mu s) \cos \left(\frac{1}{m} \cos (\mu s)\right) \quad$ and $\tau=-\frac{\mu}{m} \cos (\mu s) \sin \left(\frac{1}{m} \cos (\mu s)\right)$

Therefore

$$
f=\frac{\mu}{m} \cos (\mu s) \quad \text { and } \quad g=\frac{\mu}{m} \sin (\mu s)
$$

we have

$$
\Gamma=m \text {. }
$$

Substituting $k=\frac{\mu}{m} \cos (\mu s) \cos \left(\frac{1}{m} \cos (\mu s)\right)$ and $f=\frac{\mu}{m} \cos (\mu s)$ in the Eq.(15), we have the explicit parametric representation of such curve as follows:

$$
\left\{\begin{array}{l}
\psi_{1}(s)=\frac{n^{2} \mu}{2 m^{3}} \int\left[\int \cos (\mu s) \cos \left(\frac{1}{m} \cos (\mu s)\right)\left[\frac{n}{n+1} \sin \left(\frac{n+1}{n} \mu s\right)+\frac{n}{n-1} \sin \left(\frac{n-1}{n} \mu s\right)\right] d s\right] d s \\
\psi_{2}(s)=-\frac{n^{2} \mu}{2 m^{3}} \int\left(\int \cos (\mu s) \cos \left(\frac{1}{m} \cos (\mu s)\right)\left(\frac{n}{n+1} \cos \left(\frac{n+1}{n} \mu s\right)+\frac{n}{1-n} \cos \left(\frac{1-n}{n} \mu s\right)\right) d s\right) d s \\
\psi_{3}(s)=-\frac{n}{m} \int \cos (\mu s) \sin \left(\frac{1}{m} \cos (\mu s)\right)+m \cos \left(\frac{1}{m} \cos (\mu s)\right) d s
\end{array}\right.
$$

Example 5.2 The case of a slant-slant helix with $\kappa=\cos \left(\frac{1}{m} \sqrt{1-m^{2} s^{2}}\right)$ and $\tau=-\sin \left(\frac{1}{m} \sqrt{1-m^{2} s^{2}}\right)$.

Therefore

$$
f=1 \quad \text { and } \quad g=\frac{m s}{\sqrt{1-m^{2} s^{2}}}
$$

we have

$$
\Gamma=m \text {. }
$$

Substituting $\kappa=\cos \left(\frac{1}{m} \sqrt{1-m^{2} s^{2}}\right)=\cos \left(\frac{1}{m} \cos (n t)\right)$ and $f=1$ in the Eq.(17), we have the explicit parametric 
representation of such curve as follows:

$$
\left\{\begin{array}{l}
\psi_{1}(t)=\frac{n^{4}}{2 m^{4}} \int \cos (n t)\left[\int \cos (n t) \cos \left(\frac{1}{m} \cos (n t)\right)\left[\frac{\sin ((n+1) t)}{n+1}+\frac{\sin ((n-1) t)}{n-1}\right] d t\right] d t, \\
\psi_{2}(t)=-\frac{n^{4}}{2 m^{4}} \int \cos (n t)\left[\int \cos (n t) \cos \left(\frac{1}{m} \cos (n t)\right)\left[\frac{\cos ((n+1) t)}{n+1}+\frac{\cos ((1-n) t)}{1-n}\right] d t\right] d t, \\
\psi_{3}(t)=-\frac{n^{2}}{m^{2}} \int \cos ^{2}(n t) \sin \left(\frac{1}{m} \cos (n t)\right)+m \cos (n t) \cos \left(\frac{1}{m} \cos (n t)\right) d t
\end{array}\right.
$$

where $\theta=s$ and $t=\frac{1}{n} \arcsin (m \theta)$.

\section{Data Availability}

The data used to support the findings of this study are available the corresponding author upon request.

\section{Conflicts of Interest}

The authors declare that they have no conflicts of interest.

\section{References}

Ali, A. T. (2010). Position vectors of general helices in Euclidean 3-space. Bull. Math. Anal. Appl, 3(2), 198-205.

Ali, A. T. (2012). Position vectors of slant helices in Euclidean 3-space. Journal of the Egyptian Mathematical Society, 20(1), 1-6. https://doi.org/10.1016/j.joems.2011.12.005

Eisenhart, L. P. (1909). A treatise on the differential geometry of curves and surfaces. Ginn.

Hacisalihoglu, H. H. (2000). Differential Geometry, Ankara University, Faculty of sciences Press.

Izumiya, S., \& Takeuchi, N. (2004). New special curves and developable surfaces. Turkish Journal of Mathematics, 28(2), 153-164.

Kula, L., \& Yayli, Y. (2005). On slant helix and its spherical indicatrix. Applied Mathematics and Computation, 169(1), 600-607. https://doi.org/10.1016/j.amc.2004.09.078

Kula, L., Ekmekci, N., Yayli, Y., \& İlarslan, K. (2010). Characterizations of slant helices in Euclidean 3-space. Turkish Journal of Mathematics, 34(2), 261-274.

Lancret, M. A. (1806). Mémoire sur les courbesa double courbure. Memoires presentes alInstitut, 1, $416-454$.

Lipschutz, M. M. (1969). Schaum's outline of differential geometry. McGraw Hill Professional.

Scofield, P. D. (1995). Curves of constant precession. The American mathematical monthly, 102(6), 531-537. https://doi.org/10.1080/00029890.1995.12004613

Struik, D. J. (1961). Lectures on classical differential geometry. Courier Corporation.

Uzunoğlu, B., Gök, İ., \& Yaylı, Y. (2016). A new approach on curves of constant precession. Applied Mathematics and Computation, 275, 317-323. https://doi.org/10.1016/j.amc.2015.11.083

\section{Copyrights}

Copyright for this article is retained by the author(s), with first publication rights granted to the journal.

This is an open-access article distributed under the terms and conditions of the Creative Commons Attribution license (http://creativecommons.org/licenses/by/4.0/). 